\title{
Tradeoffs between neuromodulation and synchronized firing in affecting neuronal gain control $\mathrm{N}$ Green $* 1,2$ and Shu Chen $\mathrm{Li}^{2}$
}

\author{
Address: ${ }^{1}$ Berlin School of Mind and Brain, Humboldt Gradutate School, Humboldt University, Berlin, Germany and ${ }^{2}$ Max Planck Institute for \\ Human Development, Berlin, Germany \\ Email: N Green* - green@mpib-berlin.mpg.de \\ * Corresponding author
}

from Eighteenth Annual Computational Neuroscience Meeting: CNS*2009

Berlin, Germany. 18-23 July 2009

Published: 13 July 2009

BMC Neuroscience 2009, I0(Supp| I):PI86 doi:I0.II86/I47|-2202-I0-SI-PI86

This abstract is available from: http://www.biomedcentral.com/I47I-2202/I0/SI/PI86

(c) 2009 Green and Li; licensee BioMed Central Ltd.

\section{Introduction}

Various mechanisms of neuronal gain control (neuromodulation, synaptic, noise and neuronal synchronization) affect the stimulus-response transfer function of cortical neurons [1]. In light of the role of neurotransmitters, such as dopamine, in affecting noise in neural information processing [2], understanding how neuromodulation may interact (or tradeoff) with other mechanisms of gain control, such as neuronal synchronicity, becomes paramount $[3,4]$. Specifically, we investigate this interaction by making use of the phenomenon of stochastic resonance (SR), which states that at certain optimal levels (background) noise is beneficial for neuronal information processing $[3,5]$, thereby acting as another gain mechanism. In an early study, we showed that key characteristics of stochastic resonance (the peak of the SR function as well as the extent of the right-shift of the function) can be affected by a parameter simulating dopaminergic modulation efficacy: simulated neural networks with suboptimal neuromodulation, though still exhibiting the SR phenomena, the peak of SR is reduced, requiring more noise to arrive at the same SR effect [6]. An unresolved question is to what extent noise, its SR characteristics and systems-level neuronal information processing, such as neuronal synchronicity, are affected by deficient neuromodulation $[2,6]$.

\section{Methods}

Adapting earlier ideas $[3,4]$, we used a simplified version of a $10 \times 10$ synfire chain [7] feedforward network consist- ing of leaky integrate and fire neurons. Simulations were performed using the Neural Simulation Tool (NEST) [8]. The neurons in the first pool are stimulated externally, using Gaussian random inputs; background activity is based on Poisson process signals. In a first step, we replicated the results of $[3,4]$ showing that our network can exploit SR properties to arrive at synchronous activity. Next, to tackle the question of influence of deficient neuromodulation we simulated its effects on the network model activity by using varying sets of input spikes and their temporal precision as well as varying the degrees of background noise, effectively altering gain control. We further used Matlab to analyze the spike time data.

\section{Results and discussion}

We found that simulating deficient neuromodulation can have a significant impact on neuronal gain control. Only limited optimal (random synaptic) noise range and SR efficacy can achieve synchronous network activity. We have to formally and analytically show how neuronal synchronicity, neuromodulation and synaptic noise are exactly related. By understanding the nature and dynamics of these processes, we can obtain a valuable insight for our understanding of cognitive processing and its problems in deficient neuromodulation.

\section{Acknowledgements}

We thank M. Diesmann and T. Potjans for their valuable help on this project. 


\section{References}

I. Destexhe A, Rudolph M, Paré D: The high-conductance state of neocortical neurons in vivo. Nature Rev 2003, 4:739-75I.

2. Li S-C, Lindenberger U, Sikström S: Aging cognition: From neuromodulation to representation. Trends in Cognit Sci 200I, 5:479-486.

3. Diesmann M: Conditions for Stable Propagation of Synchronous Spiking in Cortical Networks. PhD Thesis .

4. Diesmann M, Gewaltig M-O, Aertsen A: Conditions for stable propagation of synchronous spiking in cortical neural networks. Nature 1999, 402:529-533.

5. Rouvas-Nicolis C, Nicolis G: Stochastic resonance. Scholarpedia 2007, 2: 1474.

6. $\mathrm{Li} \mathrm{S}-\mathrm{C}$, von Oertzen T, Lindenberger U: A neurocomputation model of stochastic resonance and aging. Neurocomputing 2006, 69:1553-1560.

7. Abeles M: Local Cortical Circuits: An Electrophysiological study Springer, Berlin; 1982.

8. Gewaltig M-O, Diesmann M: NEST (Neural Simulation Tool). Scholarpedia 2007, 2:1430.

Publish with Biomed Central and every scientist can read your work free of charge

"BioMed Central will be the most significant development for disseminating the results of biomedical research in our lifetime. "

Sir Paul Nurse, Cancer Research UK

Your research papers will be:

- available free of charge to the entire biomedical community

- peer reviewed and published immediately upon acceptance

- cited in PubMed and archived on PubMed Central

- yours - you keep the copyright 